\title{
Biologically effective dose (BED) of stereotactic body radiation therapy (SBRT) was an important factor of therapeutic efficacy in patients with hepatocellular carcinoma $(\leq 5 \mathrm{~cm})$
}

Jing Sun ${ }^{\dagger}$, Tao Zhang ${ }^{\dagger}$, Jia Wang, Wengang Li, Aimin Zhang, Weiping He, Dan Zhang, Dong Li, Jungiang Ding and Xuezhang Duan*

\begin{abstract}
Background: To explore the association between biologically effective dose (BED) and survival rates in Child-Pugh A classification (CP-A) small hepatocellular carcinoma (HCC) patients treated with stereotactic body radiation therapy (SBRT).

Methods: This retrospective study included 108 small HCC patients who were treated with SBRT between 2011 and 2014. The prescribed dose delivered to the tumor were 48Gy/8f, 49Gy/7f, 50Gy/5f and 54Gy/6f. The median biologically effective dose (BED 10 ) of the total prescribed dose was 100Gy (76.8-102.6Gy). Factors associated with the survival rate were examined using the Cox proportion hazards model, and the factors associated with radiation-induced liver injury (RILD) were examined by logistic regression analysis.

Results: For these patients, the median follow-up time was 42 months (6-77 months), and the 1-, 2- and 3-year overall survival (OS) rates were 96.3, 89.8 and 80.6\%, respectively. The 1-, 2- and 3-year progression-free survival (PFS) rates were $85.2,70.1$ and $60.6 \%$, respectively. The 1-, 2- and 3-year local control (LC) rates were 98.1, 96.2 and 95.1\%, respectively. The 1-, 2- and 3-year distant metastasis- free survival (DMFS) rates were 86.1, 72.8 and 61.2\%. The OS, PFS and DMFS were significantly higher in the $\mathrm{BED}_{10} \geq 100 \mathrm{~Gy}$ group than in the $\mathrm{BED}_{10}<100 \mathrm{~Gy}$ group (OS: $p=0.020$; PFS: $p=0.017$; DMFS: $p=0.012$ ). The PLT count was a predictive factor of RILD.
\end{abstract}

Conclusions: SBRT is a safe and effective option for CP-A HCC patients. A BED 10 value greater than 100Gy and lower CP score are associated with improved OS and PFS. Additionally, the peripheral PLT count are predictive factors of RILD.

Keywords: Hepatocellular carcinoma, Stereotactic body radiation therapy, Radiation-induced injury, Biologically effective dose, Platelet

\footnotetext{
* Correspondence: duanxuezhang2006@163.com

${ }^{\dagger}$ Jing Sun and Tao Zhang contributed equally to this work.

Radiation Oncology Center, The Fifth Medical Center of PLA General Hospital

(Beijing 302 Hospital), No. 100 Xi Si Huan Middle Road, Fengtai District,

Beijing 100039, China
}

(c) The Author(s). 2019 Open Access This article is distributed under the terms of the Creative Commons Attribution 4.0 International License (http://creativecommons.org/licenses/by/4.0/), which permits unrestricted use, distribution, and reproduction in any medium, provided you give appropriate credit to the original author(s) and the source, provide a link to the Creative Commons license, and indicate if changes were made. The Creative Commons Public Domain Dedication waiver (http://creativecommons.org/publicdomain/zero/1.0/) applies to the data made available in this article, unless otherwise stated. 


\section{Background}

Hepatocellular carcinoma (HCC) is the sixth most common malignancy and the third most common cause of cancer mortality worldwide, and only approximately 20$30 \%$ of patients with $\mathrm{HCC}$ are eligible for surgical treatment, including liver resection and liver transplantation $[1,2]$. Accumulating data have shown that stereotactic body radiation therapy (SBRT) is a safe and effective treatment for $\mathrm{HCC}$, especially in patients with inoperable or recurrent $\mathrm{HCC}$ [3-5]. Furthermore, Su et al. [6] compared the efficacy of stereotactic ablative radiation therapy (SABR) versus liver resection for treating small HCC $(<5 \mathrm{~cm})$ patients with Child-Pugh class A (CP-A) cirrhosis and concluded that SABR has local effects that are similar to those of liver resection. Wahl et al. [7] reported that SBRT and radiofrequency ablation (RFA) were equally effective for treating small HCCs. We conducted this retrospective study to evaluate the efficacy of SBRT and identify prognostic factors related to the efficacy of SBRT in patients with HCC.

In other cancers, such as lung and cervical cancers, with the delivery of increasing biologically effective doses (BEDs) to lesions, the OS of patients increased [8-10]. However, due to the scarcity of data on HCC, the relationship between the BEDs and the efficacy of SBRT in HCC patients was included in our study.

Radiation-induced liver injury (RILD) is often a fatal complication of SBRT and should be avoided in patients with HCC; furthermore, the CP classification is an important predictive factor of RILD [11-13]. Therefore, we investigated the incidence of RILD in the included patients and searched for predictive factors among their clinical data, including biochemical parameters and the peripheral platelet (PLT) and white blood cell (WBC) counts.

\section{Methods}

\section{Enrolled patients' characteristics and SBRT parameters}

We conducted a retrospective observation of CP-A HCC patients. The eligibility criteria were the following: (a) primary HCC diagnosed by a surgeon and/or radiologist and oncologist according to the international guidelines for the management of HCC or by pathology [14]; (b) single lesion and longest tumor diameter $<5.0 \mathrm{~cm}$; (c) CP-A classification; (d) Eastern Cooperative Oncology Group (ECOG) score 0-1; (e) distances between tumor and normal organs (esophagus, stomach, duodenum, bowel) were more than $5 \mathrm{~mm}$; (f) unsuitable for other therapies, such as patients with heart disease, uncontrolled diabetes, uncontrolled hypertension, etc. (g) rejecting other therapies such as resection, liver transplantation, etc. (h) platelet count $\geq 50 \times 10^{9} / \mathrm{L}$, white blood count $\geq 1.5 \times 10^{9} / \mathrm{L}$ and (i) patients infected with hepatitis B virus who were treated with adefovir or entecavir; patients infected with hepatitis $C$ virus whose $\mathrm{HCV}$ DNA were negative. The exclusion
Table 1 Clinical, biochemical characteristics and radiation planning parameters of patients enrolled in this study

\begin{tabular}{|c|c|}
\hline Variables & $\mathrm{n}$ \\
\hline \multicolumn{2}{|c|}{ Clinical and biochemical characteristics } \\
\hline \multicolumn{2}{|l|}{ Sex } \\
\hline Male & $80(74.07 \%)$ \\
\hline Female & $28(25.93 \%)$ \\
\hline \multicolumn{2}{|l|}{ Age (years) } \\
\hline Median & 54 \\
\hline Range & $37-77$ \\
\hline \multicolumn{2}{|l|}{ Underlying liver disease } \\
\hline Hepatitis B & 97 (89.81\%) \\
\hline Hepatitis C & $7(6.48 \%)$ \\
\hline Alcoholic hepatitis & $3(2.78 \%)$ \\
\hline None & $1(0.93 \%)$ \\
\hline \multicolumn{2}{|c|}{ Maximum tumor diameter $(\mathrm{cm})$} \\
\hline Median & 2.3 \\
\hline Range & $0.7-4.9$ \\
\hline \multicolumn{2}{|l|}{$\operatorname{AFP}(\mathrm{ng} / \mathrm{ml})^{a}$} \\
\hline Median & 33.13 \\
\hline Range & $0.8-7896$ \\
\hline \multicolumn{2}{|l|}{ Child-Pugh score } \\
\hline 5 & 97 (89.81\%) \\
\hline 6 & $11(10.19 \%)$ \\
\hline \multicolumn{2}{|l|}{ ALBI grade } \\
\hline Grade 1 & $34(31.48 \%)$ \\
\hline Grade 2 & $74(68.52 \%)$ \\
\hline \multicolumn{2}{|l|}{ WBC count $\left(\times 10^{9} / L\right)$} \\
\hline Median & 4.86 \\
\hline Range & $1.53-9.4$ \\
\hline \multicolumn{2}{|l|}{ PLT count $\left(\times 10^{9} / \mathrm{L}\right)$} \\
\hline Median & 118 \\
\hline Range & $50-283$ \\
\hline \multicolumn{2}{|c|}{ Radiation planning parameters } \\
\hline \multicolumn{2}{|c|}{ Isodose line of maximum dose } \\
\hline Median (range, \%) & $72(60-86)$ \\
\hline \multicolumn{2}{|c|}{ Residual normal liver volume } \\
\hline Median (range, $\mathrm{cm}^{3}$ ) & $1324(834-2493)$ \\
\hline \multicolumn{2}{|c|}{ Mean dose of whole liver volume minus GTV } \\
\hline Median (range, Gy) & $9.07(4.04-12.05)$ \\
\hline \multicolumn{2}{|c|}{ Dose received by volume of liver } \\
\hline D700 & $4.99(1.40-13.50)$ \\
\hline \multicolumn{2}{|c|}{$\mathrm{BED}_{10}^{\mathrm{b}}$ of Plan target volume (PTV) } \\
\hline Median (range, Gy) & $100(76.8-102.6)$ \\
\hline
\end{tabular}


criteria were the following: (a) tumor thrombus; (b) lymph node involvement; and (c) extrahepatic metastasis. Patients were treated with SBRT at The Fifth Medical Center of PLA General Hospital between 2011 and 2014. They did not receive any other treatments, such as RFA or transcatheter arterial chemoembolization (TACE) before enrollment into our study. All patients were managed in multidisciplinary setting with all legitimate treatment options available and provided with written informed consent before treatment.

The baseline data of the 108 patients in our study are listed in Table 1.ALBI parameter in this study was calculated from baseline blood work according to Johnson et al. [15]. ALBI $=\left[\log _{10}\right.$ bilirubin $\left.\times 0.66\right]+[$ albumin $\times(-0.085)]$, where bilirubin is in $\mu \mathrm{mol} / \mathrm{L}$ and albumin is in $\mathrm{g} / \mathrm{L}$; the cutoffs were used to assign each patient to one of three prognostic groups indicating the ALBI grade (range, 1 to 3 ). The cutoffs were as follows: $x b \leq-2.60$ (ALBI grade 1), >2.60 to $\leq 1.39$ (ALBI grade 2), and $>-1.39$ (ALBI grade 3).

\section{Radiation treatment technique}

All enrolled patients underwent the implantation of 4 to 6 fiducials one week prior to SBRT (CyberKnife, Accuray, USA). All plans were designed using G4 CyberKnife MultiPlan (version 4.0.2) and were applied with dynamic respiration tracking combined with fiducial tracking.

An oncologist contoured the gross tumor volume (GTV) and organs at risk (normal liver, kidneys, esophagus, stomach, duodenum, bowel and spinal cord). The planning target volume (PTV) was expanded 3-5 mm around the GTV, which contoured $100 \%$ of GTV. The prescribed dose delivered to the tumor were $48 \mathrm{~Gy} / 8 \mathrm{f}$, $49 \mathrm{~Gy} / 7 \mathrm{f}, 50 \mathrm{~Gy} / 5 \mathrm{f}$ and $54 \mathrm{~Gy} / 6 \mathrm{f}$. The selection of dose depended on the relation between lesion and bile duct.
If the distance between tumor and bile duct was less than $3 \mathrm{~mm}$, the prescribed dose delivered to the tumor were $48 \mathrm{~Gy} / 8 \mathrm{f}$ or $49 \mathrm{~Gy} / 7 \mathrm{f}$. The normal tissue dose was within the normal radiotherapy tolerance dose (TG-101) [16]. In this study, both the single dose and the total dose varied, so the BED was chosen as the parameter of reaction dose fractionated schemes.

The BED was calculated according to the value of $\alpha / \beta$ (10Gy, BED 10$)$ using the formula BED $=\mathrm{D}(1+\mathrm{d} /[\alpha / \beta])$ $[17,18]$, where $\mathrm{D}$ is the total dose delivered, and $\mathrm{d}$ is the dose per fraction. The median $\mathrm{BED}_{10}$ of the total prescribed dose was 100Gy (76.8-102.6Gy) in our research.

\section{Follow-up study}

All patients underwent a liver function assessment and routine blood examinations before treatment. After SBRT, the patients were followed every 3 months for 1 year and every 6 months thereafter until June 2018.

\section{Toxicity evaluation}

Radiation-related toxicity was measured based on Toxicity criteria of the Radiation Therapy Oncology Group (RTOG) and the European organization for research and treatment of cancer (EORTC) [19].

The liver toxicity reaction evaluation was based on the definition of RILD, of which there are two types: classic RILD and non-classic RILD.

Classic RILD usually manifests as symptoms of fatigue, hepatomegaly, and anicteric ascites, etc., 1-3 months after radiotherapy. Moreover, the serum alkaline phosphatase (ALP) level in these patients increases to more than twice the normal level, while the serum transaminase and bilirubin levels in remain normal [20,21].

\begin{tabular}{|c|c|c|c|}
\hline \multirow{12}{*}{$\begin{array}{l}\text { Tumor recurrence and } \\
\text { metastasis }\end{array}$} & \multirow[t]{3}{*}{ Liver metastasis in 36 patients } & \multicolumn{2}{|l|}{16 patients treated with SBRT } \\
\hline & & \multicolumn{2}{|l|}{12 patients treated with targeted therapy } \\
\hline & & \multicolumn{2}{|l|}{8 patients treated conservatively } \\
\hline & \multirow[t]{2}{*}{ Lymph node metastasis in 2 patients } & \multicolumn{2}{|l|}{1 patient treated with SBRT } \\
\hline & & \multicolumn{2}{|l|}{1 patient treated conservatively } \\
\hline & Bone metastasis in 1 patient & \multicolumn{2}{|l|}{ Treated with SBRT } \\
\hline & Brain metastasis in 1 patient & \multicolumn{2}{|l|}{ Treated with conservatively } \\
\hline & Lung metastasis in 1 patient & \multicolumn{2}{|l|}{ Treated with SBRT } \\
\hline & \multirow{4}{*}{ Multiple organ metastasis in 4 patients } & Lymph node and lung in 1 patient & Treated with targeted therapy \\
\hline & & Liver and lung in 1 patient & Treated conservatively \\
\hline & & Liver, lymph node and brain in 1 patient & Treated with targeted therapy \\
\hline & & Liver. bone and lung in a patient & Treated conservatively \\
\hline
\end{tabular}


Non-classic RILD occurs in patients with underlying chronic hepatic diseases, who suffer from jaundice and/ or remarkably elevated serum transaminase levels (increased by more than fivefold compared to normal levels) [22, 23].

Meanwhile, CP score progression (increasing by 2 or more scores) was also a clinical metric for non-classic RILD [24] .

\section{Tumor recurrence and treatment}

When the recurrence/metastasis was confirmed, secondline treatments were individualized according to the number and location of the recurrent tumors and the liver function status, while considering the patient's preferences. Therapeutic options included repeated SBRT, targeted therapy and conservative treatment.

\section{Statistical analysis}

OS was calculated starting from the date of SBRT to the date of the final follow-up or demise of the patients. PFS was estimated starting from the date of SBRT to the date of disease progression or patient death. LC was defined starting from the date of SBRT to the date of treated-lesion progression or patient death. DMFS was defined starting from the date of SBRT to the date of distant metastasis occurrence (out-field relapse). OS, PFS, LC and DMFS were estimated using the Kaplan-Meier method. OS-, PFS-, LC- and DMFS- related group analyses of $\mathrm{BED}_{10}$ were performed using the log rank test. Univariate and multivariable hazard ratios were calculated using the Cox proportion hazard model. A binary logistic regression method was employed to investigate each prognostic factor of RILD. Variables with $p$-values less than 0.2 in the univariate analysis were included in the multivariate analysis with forward selection. For comparisons between the baseline variables, the $x^{2}$ test and Fisher's exact test were performed.

All statistical analyses were performed using SPSS 22.0 software (IBM) and STATA 15.0 software. $P$ values < 0.05 were considered statistically significant.

\section{Results}

The median follow-up period was 42 months (range, 677 months). By June 2018, 45 patients had experienced relapse or metastasis; 36 patients experienced liver metastasis, 2 experienced lymph node metastasis, 1 experienced bone metastasis, 1 experienced brain metastasis, 1 experienced lung metastasis and 4 experienced multiple organ metastases. The treatment was ultimately selected by the patient (Fig. 1).

\section{Survival outcomes}

After 6 months of SBRT, there were 65 patients with CR (60.19\%), 30 patients with PR (27.78\%), 4 patients with SD
A

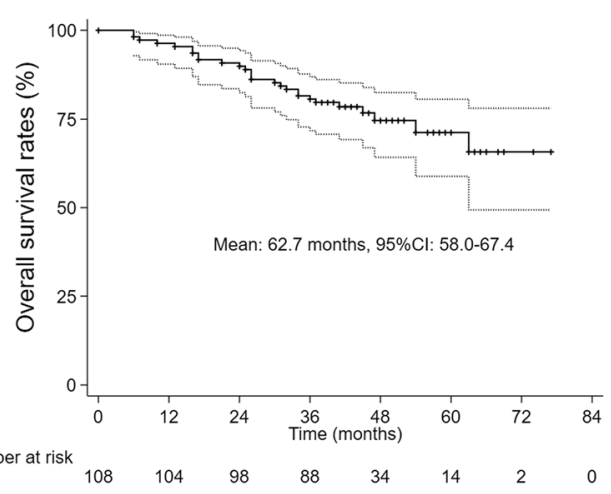

B

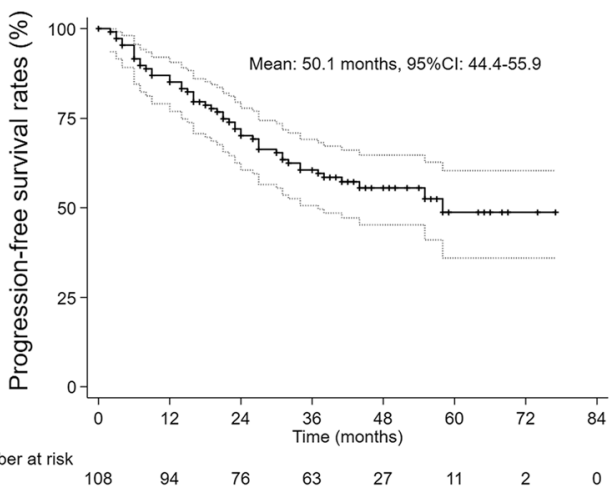

C
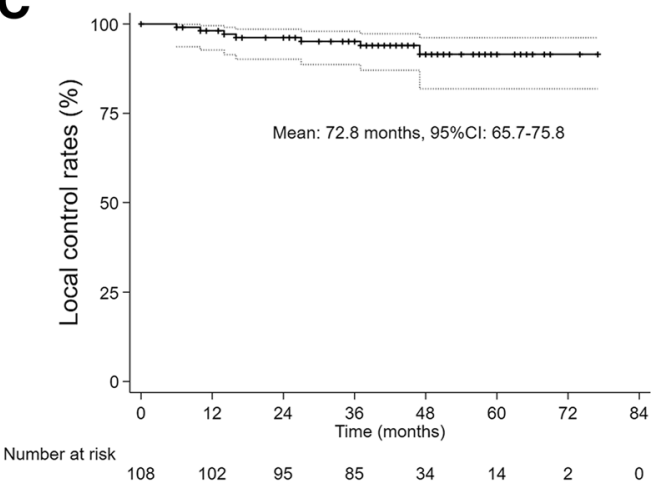

D

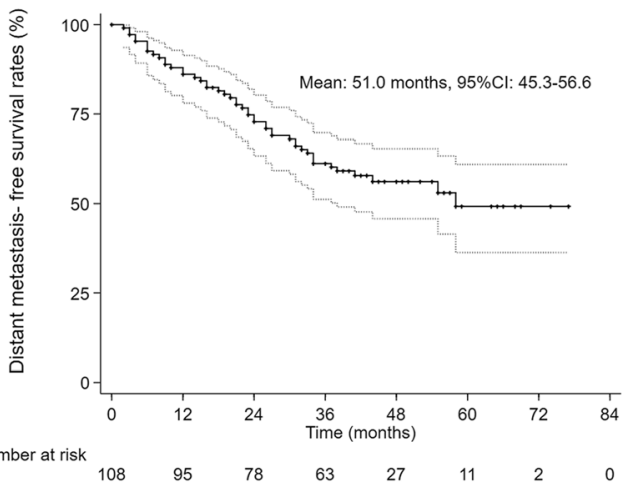

Fig. 2 Kaplan-Meier curve of overall survival (a), progression-free survival (b), local control (c) and distant metastasis-free survival (d) 
Table 2 Univariate and multivariate Cox regression analysis of OS

\begin{tabular}{|c|c|c|c|c|}
\hline \multirow[b]{2}{*}{ Patient details } & \multicolumn{2}{|c|}{ Univariate Cox regression } & \multicolumn{2}{|c|}{ Multivariate Cox regression } \\
\hline & $p$ value & Hazard ratio $(95 \% \mathrm{Cl})$ & $p$ value & Hazard ratio $(95 \% \mathrm{Cl})$ \\
\hline Sex (male/female) & 0.214 & $1.856(0.700-4.922)$ & & \\
\hline Age & 0.983 & $1.000(0.960-1.043)$ & & \\
\hline Hepatitis type (B/C/alcoholic/others) & 0.645 & $1.167(0.606-2.247)$ & & \\
\hline Maximum tumor diameter & 0.368 & $1.222(0.790-1.889)$ & & \\
\hline ECOG PS & 0.432 & $1.621(0.486-5.402)$ & & \\
\hline AFP & 0.968 & $1.000(1.000-1.000)$ & & \\
\hline Child-Pugh score (5/6) & 0.095 & $2.300(0.866-6.108)$ & 0.048 & $2.740(1.011-7.425)$ \\
\hline ALBI grade & 0.708 & $1.156(0.542-2.465)$ & & \\
\hline WBC count & 0.181 & $0.822(0.618-1.095)$ & & \\
\hline PLT count & 0.134 & $0.993(0.984-1.002)$ & & \\
\hline Residual normal liver volume & 0.375 & $0.999(0.997-1.001)$ & & \\
\hline Mean dose of whole liver volume minus GTV & 0.810 & $0.997(0.972-1.022)$ & & \\
\hline D700 & 0.252 & $1.151(0.905-1.463)$ & & \\
\hline $\mathrm{BED}_{10}$ of PTV & 0.030 & $0.960(0.925-0.996)$ & 0.017 & 0.955 (0.919-0.992) \\
\hline
\end{tabular}

(3.70\%) and 9 patients with $\mathrm{PD}(8.33 \%)$. The response rate was $(\mathrm{CR}+\mathrm{PR}) / 108 \times 100 \%=87.96 \%$, and the disease control rate was $(\mathrm{CR}+\mathrm{PR}+\mathrm{SD}) / 108 \times 100 \%=$ 91.67\%.

By June 2018, 27 patients died: 6 patients died of hepatic failure; 6 died of upper gastrointestinal bleeding; 1 died of hepatorenal syndrome; 1 died of infectious shock; 1 died of pulmonary or brain metastasis complications; and 12 died of unknown causes.

The 1-, 2- and 3-year OS rates were 96.3, 89.8 and $80.6 \%$, respectively (Fig. 2a). The 1-, 2- and 3-year
PFS rates were 85.2, 70.1 and $60.6 \%$, respectively (Fig. 2b). The 1-, 2- and 3-year LC rates were 98.1, 96.2 and $95.1 \%$, respectively (Fig. 2c).

The 1-, 2- and 3-year DMFS rates were 86.1, 72.8 and $61.2 \%$, respectively (Fig. 2d). The 5 -year cumulative OS, PFS, LC and DMFS were 71.2,48.7,91.5 and 49.2\%, respectively.

In the Cox proportional hazard model, the Child-Pugh score and $\mathrm{BED}_{10}$ were independent prognostic factors of OS (Table 2), PFS (Table 3) and DMFS (Table 4) on multivariate analysis.

Table 3 Univariate and multivariate Cox regression analysis of PFS

\begin{tabular}{|c|c|c|c|c|}
\hline \multirow[b]{2}{*}{ Patient details } & \multicolumn{2}{|c|}{ Univariate Cox regression } & \multicolumn{2}{|c|}{ Multivariate Cox regression } \\
\hline & $p$ value & Hazard ratio $(95 \% \mathrm{Cl})$ & $p$ value & Hazard ratio $(95 \% \mathrm{Cl})$ \\
\hline Sex (male/female) & 0.094 & $1.864(0.900-3.858)$ & & \\
\hline Age & 0.673 & $0.993(0.962-1.025)$ & & \\
\hline Hepatitis type (B/C/alcoholic/others) & 0.043 & $1.546(1.013-2.359)$ & & \\
\hline Maximum tumor diameter & 0.848 & $1.026(0.789-1.334)$ & & \\
\hline ECOG PS & 0.388 & $1.505(0.595-3.805)$ & & \\
\hline AFP & 0.440 & $1.000(1.000-1.000)$ & & \\
\hline Child-Pugh (5/6) & 0.071 & $2.100(0.938-4.071)$ & 0.029 & $2.500(1.096-5.705)$ \\
\hline ALBI grade & 0.156 & $0.656(0.367-1.174)$ & & \\
\hline WBC count & 0.051 & $0.850(0.723-1.001)$ & & \\
\hline PLT count & 0.997 & $0.993(0.992-1.003)$ & & \\
\hline Residual normal liver volume & 0.375 & $0.999(0.997-1.001)$ & & \\
\hline Mean dose of whole liver volume minus GTV & 0.524 & $0.959(0.845-1.090)$ & & \\
\hline D700 & 0.839 & $1.018(0.857-1.210)$ & & \\
\hline BED $_{10}$ of PTV & 0.020 & $2.063(1.120-3.803)$ & 0.020 & $2.063(1.120-3.803)$ \\
\hline
\end{tabular}


Table 4 Univariate and multivariate Cox regression analysis of DMFS

\begin{tabular}{|c|c|c|c|c|}
\hline \multirow[b]{2}{*}{ Patient details } & \multicolumn{2}{|c|}{ Univariate Cox regression } & \multicolumn{2}{|c|}{ Multivariate Cox regression } \\
\hline & $p$ value & Hazard ratio $(95 \% \mathrm{Cl})$ & $p$ value & Hazard ratio $(95 \% \mathrm{Cl})$ \\
\hline Sex (male/female) & 0.114 & $1.801(0.868-3.736)$ & & \\
\hline Age & 0.777 & $0.995(0.964-1.028)$ & & \\
\hline Hepatitis type (B/C/alcoholic/others) & 0.080 & $1.668(0.940-1.028)$ & & \\
\hline Maximum tumor diameter & 0.901 & $1.017(0.780-1.326)$ & & \\
\hline ECOG & 0.220 & $1.799(0.704-4.594)$ & & \\
\hline AFP & 0.471 & $1.000(1.000-1.000)$ & & \\
\hline Child-Pugh score (5/6) & 0.054 & $2.214(0.996-4.965)$ & 0.021 & $2.653(1.160-6.067)$ \\
\hline ALBI grade & 0.487 & $0.714(0.277-1.843)$ & & \\
\hline WBC count & 0.061 & $0.854(0.724-1.007)$ & & \\
\hline PLT count & 0.320 & $0.997(0.992-1.003)$ & & \\
\hline Residual normal liver volume & 0.982 & $1.000(0.998-1.002)$ & & \\
\hline Mean dose of whole liver volume minus GTV & 0.898 & $0.993(0.886-1.112)$ & & \\
\hline D700 & 0.496 & $1.064(0.889-1.274)$ & & \\
\hline $\mathrm{BED}_{10}$ of PTV & 0.013 & $0.964(0.937-0.992)$ & 0.006 & $0.959(0.932-0.988)$ \\
\hline
\end{tabular}

Comparison between $\mathrm{BED}_{10} \geq 100 \mathrm{~Gy}$ and $\mathrm{BED}_{10}<100 \mathrm{~Gy}$

To further examine the $\mathrm{BED}_{10}$, we divided the patients into two groups with a $\mathrm{BED}_{10}$ of $100 \mathrm{~Gy}$ as the cutoff, i.e., the $\mathrm{BED}_{10} \geq 100 \mathrm{~Gy}$ and $\mathrm{BED}_{10}<100 \mathrm{~Gy}$ groups. There were no differences in the detail of the patients between the two groups (Table 5). There were 84 patients in the $\mathrm{BED}_{10} \geq 100 \mathrm{~Gy}$ group and 24 patients in the $\mathrm{BED}_{10}<100 \mathrm{~Gy}$ group. The OS, PFS and DMFS rates were significantly higher in the $\mathrm{BED}_{10} \geq 100 \mathrm{~Gy}$ group than in the $\mathrm{BED}_{10}<100 \mathrm{~Gy}$ group (OS: $p=0.020$, Fig. 3a; PFS: $p=0.017$, Fig. 3b; DMFS: $p=0.012$, Fig. $3 \mathrm{~d}$ ). However, there were no significant differences in LC between the two groups $(p=0.409$, Fig. 3c).

\section{Toxicity outcomes}

All 108 patients finished the SBRT treatments. Grade 12 acute toxicity reactions occurred in 32 patients, including abdominal pain ( 3 patients in $\mathrm{BED}_{10} \geq 100 \mathrm{~Gy}$ group, 1 patients in $\mathrm{BED}_{10}<100$ Gy group, $p=0.89$ ), fatigue (13 patients in $\mathrm{BED}_{10} \geq 100 \mathrm{~Gy}$ group, 5 patients in $\mathrm{BED}_{10}<$ 100Gy group, $p=0.53$ ), vomiting and anorexia (22 patients were in $\mathrm{BED}_{10} \geq 100 \mathrm{~Gy}$ group, 6 patients were in BED $_{10}<100$ Gy group, $p=0.92$ ), which could be relieved gradually by symptomatic treatment.

\section{Liver toxicity}

The most serious complication of RILD is the liver failure-resulted death. We reviewed the causes of death of

Table 5 Details of the patients in the two groups

\begin{tabular}{llll}
\hline Details & BED $_{10} \geq 100$ Gy & BED $_{10}<100$ Gy & $p$ value \\
\hline Sex (male/female) & & $18 / 6$ & 0.907 \\
Age & $62 / 22$ & $54.60 \pm 11.59$ & 0.885 \\
Maximum tumor diameter & $54.33 \pm 8.82$ & $2.74 \pm 1.10$ & 0.406 \\
ECOG PS (0/1) & $2.50 \pm 1.02$ & $22 / 2$ & 0.844 \\
Child-Pugh score (5/6) & $78 / 6$ & $22 / 2$ & 0.734 \\
ALBl grade (1/2) & $75 / 9$ & $9 / 15$ & 0.472 \\
WBC count & $25 / 59$ & $4.68 \pm 1.51$ & 0.528 \\
PLT count & $5.00 \pm 1.99$ & $112.04 \pm 39.47$ & 0.244 \\
Residual normal liver volume & $127.06 \pm 59.06$ & $1390.36 \pm 329.11$ & 0.614 \\
D700 & $1345.33 \pm 286.81$ & $4.41 \pm 3.17$ & 0.087 \\
Percent of PTV volume enclosed by isodose line (\%) & $5.50 \pm 2.27$ & $91.53 \pm 1.77$ & 0.280 \\
\hline
\end{tabular}




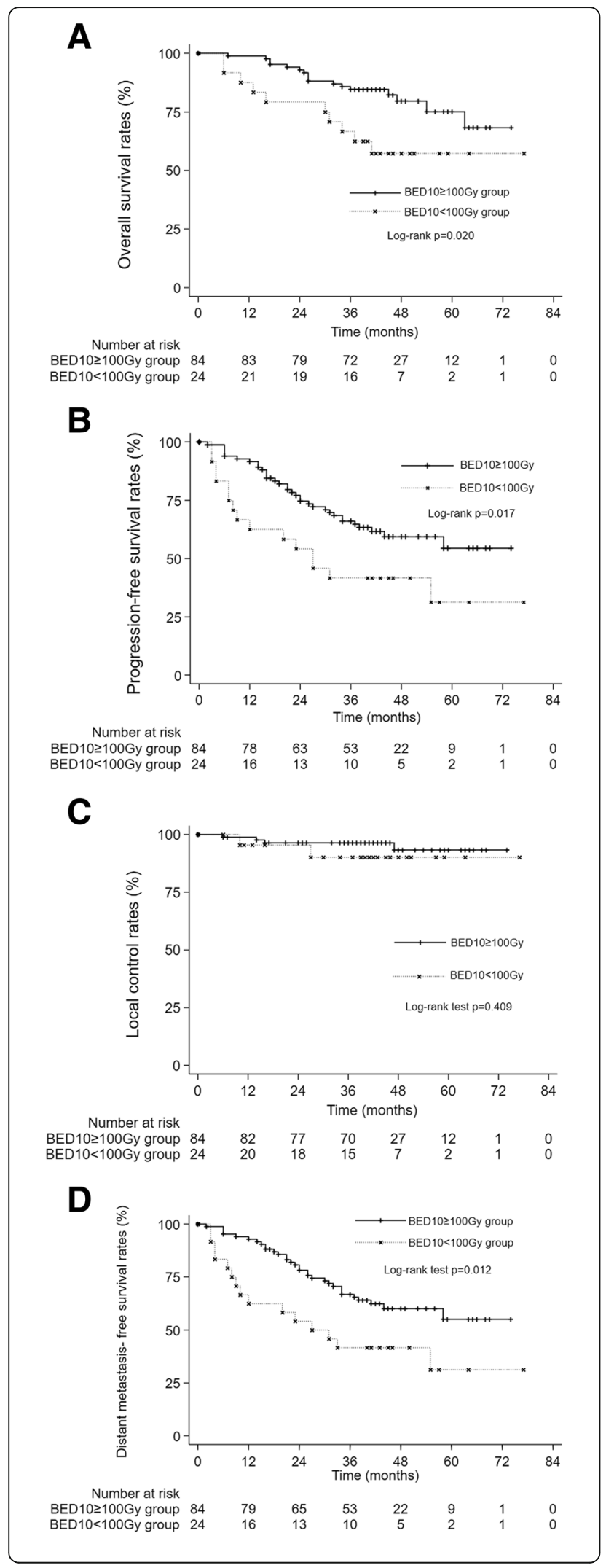

Fig. $3 \mathrm{~A}$ OS in the $\mathrm{BED}_{10} \geq 100 \mathrm{~Gy}$ and $\mathrm{BED}_{10}<100 \mathrm{~Gy}$ groups. The $1-, 2$ - and 3-year OS rates were $98.8,92.9$ and $84.5 \%$ in the BED $_{10} \geq 100$ Gy group and $87.5,79.2$ and $66.7 \%$ in the BED $_{10}<$ 100 Gy group, respectively $(p=0.020)$. B PFS in the BED $10 \geq 100$ Gy and $\mathrm{BED}_{10}<100$ Gy groups. The 1-, 2- and 3-year PFS rates were $91.7,74.7$ and $66.0 \%$ in the $\mathrm{BED}_{10} \geq 100$ Gy group and 62.5 , 54.2 and $41.7 \%$ in the $\mathrm{BED}_{10}<100$ Gy group, respectively $(p=0$.

017). C LC in the BED $10 \geq 100 \mathrm{~Gy}$ and $\mathrm{BED}_{10}<100 \mathrm{~Gy}$ groups. The 1 -, 2- and 3-year $L C$ rates were $98.8,96.4$ and $96.4 \%$ in the $B D_{10} \geq 100$ Gy group and $95.5,95.5$ and $90.2 \%$ in the $\mathrm{BED}_{10}<100$ Gy group, respectively $(p=0.409)$. D DMFS in the $\mathrm{BED}_{10} \geq 100 \mathrm{~Gy}$ and $\mathrm{BED}_{10}<100$ Gy groups. The 1-, 2- and 3-year DMFS rates were 92.9, 80.7 and 66.9\% in the $\mathrm{BED}_{10} \geq 100$ Gy group and 62.5, 54.2 and $41.7 \%$ in the $\mathrm{BED}_{10}<100$ Gy group, respectively $(p=0.012)$

four patients who died within a year after receiving SBRT, among which only one died from liver failure. The patient' tumor progressed three months after treatment, but his liver function remained normal. The patient only received conservative treatment afterwards. Therefore, we contributed the death of this patient to tumor progression instead of RILD.

In our research, eight patients were diagnosed with RILD through laboratory tests; 4 patients showed classic RILD, and 4 patients showed non-classic RILD (The parameters of RILD patients were shown in Table 6). Among these patients, 1 patient $(1 / 24)$ was in $\mathrm{BED}_{10}<$ 100Gy group while 7 patients $(7 / 84)$ were in $\mathrm{BED}_{10} \geq$ 100Gy group $(p=0.49)$. The condition of all patients who were diagnosed with RILD was relieved after symptomatic treatment, and none of these patients died. The analysis of the factors influencing RILD is shown in Table 7. A lower PLT count was associated with an increased risk of RILD on multivariate analysis.

\section{Discussion}

In this study, we retrospectively reviewed a large group of CP-A HCC patients treated with SBRT over a threeyear span as an extended analysis of our previous observations [25]. Compared to prior studies, the OS, PFS, LC and DMFS rates were all satisfactory in this study. Su et al. [4] reported 3- and 5-year OS rates of 73.5 and $64.3 \%$ and 3- and 5-year PFS rates of 58.3 and 36.4\%, respectively, with doses of 42-46Gy administered in 3-5 fractions and single-fraction doses of 28-30 Gy. Among 77 HCC patients treated with SBRT, Andres et al. [3] found 1- and 2-year OS rates of 81.8 and $56.6 \%$, respectively, and 1- and 2-year LC rates of $99 \%$. The total dose of 45 Gy in 3 fractions was prescribed to the $80 \%$ isodose line. Their OS rates were lower than ours, which have been caused by the inclusion of CP-B patients and/or patients with previous treatments. This finding was confirmed by their conclusion that the CPB classification was associated with a poor prognosis. Moreover, previous treatments may also be an affect 
Table 6 The parameters of RILD patients before and after SBRT

\begin{tabular}{|c|c|c|c|c|c|c|c|c|c|c|c|c|}
\hline & \multicolumn{2}{|l|}{ ALP } & \multicolumn{2}{|c|}{ Bilirubin } & \multicolumn{2}{|l|}{$\mathrm{ALT}$} & \multicolumn{2}{|l|}{ AST } & \multicolumn{2}{|l|}{ ALB } & \multicolumn{2}{|c|}{ Ascites } \\
\hline & Pre- & Post- & Pre- & Post- & Pre- & Post- & Pre- & Post- & Pre- & Post- & Pre- & Post- \\
\hline \multirow[t]{4}{*}{ Classic RILD } & 131 & 341 & 9.8 & 9.5 & 28 & 39 & 32 & 32 & 43 & 36 & - & - \\
\hline & 67 & 80 & 12.8 & 9.9 & 43 & 34 & 43 & 29 & 46 & 35 & - & ++ \\
\hline & 59 & 327 & 14.5 & 8.4 & 13 & 18 & 16 & 29 & 44 & 41 & - & + \\
\hline & 113 & 362 & 7.8 & 8.2 & 16 & 31 & 46 & 38 & 42 & 44 & - & - \\
\hline \multirow[t]{4}{*}{ Non-Classic RILD } & 67 & 136 & 22.7 & 51.6 & 30 & 608 & 28 & 363 & 36 & 40 & - & - \\
\hline & 79 & 81 & 18.5 & 35.6 & 28 & 17 & 37 & 42 & 39 & 34 & - & - \\
\hline & 79 & 116 & 12 & 16 & 22 & 214 & 27 & 223 & 34 & 33 & - & - \\
\hline & 73 & 147 & 26.1 & 51.4 & 34 & 749 & 42 & 678 & 36 & 32 & - & + \\
\hline
\end{tabular}

factor. Ueno et al. [26] reviewed 296 patients with single nodular $\mathrm{HCC} \leq 5 \mathrm{~cm}$ with Child-Pugh A between 2001 and 2011 who underwent surgical resection (SR, $n=136$ ) and radiofrequency thermal ablation (RFA, $n=$ 160 ), and they found that 5-year OS rates of SR and RFA among all patients were 70.1 and $69.8 \%$, respectively $(P=0.14)$. The 5 -year OS was similar to the 5 -year cumulative $\mathrm{OS}$ in our result.

$\mathrm{CP}$ score was an influential factor in OS and PFS in our study, which may be related to the extent to which $\mathrm{CP}$ reflects cirrhosis, which is associated with occurrence of complication and tumorigenesis. Moreover, we found that the OS, PFS and DMFS rates also increased significantly with $\mathrm{BED}_{10} \geq 100 \mathrm{~Gy}$ in treating $\mathrm{HCC}$ with SBRT, but with no significant difference in the LC rates. The correlation between LC and BEDs in our study was consistent with Ohri' findings [27], but he didn't explore the relation between BEDs and survival rates. In addition to initial radiotherapy, the efficacy of subsequent treatment after recurrence was also a factor affecting OS. The relation between PFS and BEDs of our study, we speculate that SBRT could change immune microenvironment, which can be proven by DMFS. This assumption is also supported by some other tumor-related articles. Lee et al. [28] reported that ablative radiotherapy dramatically increased T-cell priming in draining lymphoid tissues, leading to reduction/eradication of the primary tumor or distant metastases. Meanwhile, antitumor immunity significantly contributes to the superior response induced by one dose of $20 \mathrm{~Gy}$ compared with that induced by 4 doses of 5Gy. Schaue et al. [29] studied the tumor-specific immune response in mice with murine melanoma irradiated with 15Gy administered in different fractionated dose schemes, and the results showed that a single dose of $7.5 \mathrm{~Gy}$ or higher, but not lower than $5 \mathrm{~Gy}$, was immune-stimulatory, as mediated by tumor-reactive $\mathrm{T}$ cells. They all showed that under the condition that the total dose remained unchanged, the superior anti-tumor immune response was related with the higher single dose. And it is the same with BEDs, which increase with higher single dose when the total dose remained the same. Therefore, with higher BEDs causing a better anti-

Table 7 Univariate and multivariate logistic regression analysis of RILD

\begin{tabular}{lllll}
\hline & \multicolumn{2}{l}{ Univariate logistic regression } & & Multivariate logistic regression \\
\cline { 2 - 4 } \cline { 3 - 4 } Patient details & $p$ value & Hazard ratio $(95 \% \mathrm{Cl})$ & & Hazard ratio $(95 \% \mathrm{Cl})$ \\
\hline Sex (male/female) & 0.116 & $0.304(0.069-1.345)$ & \\
Age & 0.793 & $0.542(0.890-1.058)$ & \\
Hepatitis type (B/C/alcoholic/others) & 0.096 & $0.019(0.309-3.269)$ & \\
Maximum tumor diameter & 0.015 & $0.096(0.531-2.092)$ & \\
Child-Pugh score (5/6) & 0.999 & 0.000 & \\
ALBI grade & 0.398 & $0.523(0.116-2.352)$ & 0.034 \\
WBC count & 0.009 & $0.422(0.220-0.808)$ & \\
PLT count & 0.006 & $0.966(0.942-0.990)$ & \\
Residual normal liver volume & 0.973 & $1.001(0.999-1.004)$ & \\
Mean dose of whole liver volume minus GTV & 0.821 & $0.997(0.972-1.024)$ & \\
D700 & 0.002 & $0.029(0.901-1.695)$ & \\
BED 10 of PTV & 0.696 & $1.018(0.930-1.116)$ & \\
\hline
\end{tabular}


tumor immune microenvironment, the recurrence rates of tumor decreased. However, it's only our hypothesis. In our other studies, we found that in these patients different $\mathrm{BED}_{10}$ values lead to different changes of immune system, such as NK cell functions, and further experiments are in progress (data not shown).

The influential factor of RILD is the pretreatment PLT count, as demonstrated by Velec et al. [11]. Some studies shown that the PLT count indirectly reflects the degree of liver cirrhosis by indicating the degree of portal hypertension and hypersplenism [30, 31]. Nozaki et al. [32] performed in vitro and in vivo studies, and they have proven that thrombopoietin promotes liver regeneration and improves liver cirrhosis by increasing the PLT level, indirectly implying that PLT decrease would worsen liver cirrhosis. We think it is the relation between the PLT count and liver cirrhosis, an influential factor of RILD that makes PLT count an influential factor of RILD [33]. In clinical work, we found Child-Pugh score and PLT were not compatible. Degree of liver cirrhosis could affect liver nutrition supply by affecting liver hemodynamics, which may affect liver function. However, liver cirrhosis degree is not the only influence factor of liver function, which may be affected by many other factors, including effective liver volume, hepatic cell function and compensate ability of liver, etc. Moreover, PLT is not a parameter affecting Child-Pugh score. Our result reminds us that besides Child-Pugh score, PLT is also a non-negligible factor.

Though the limited dose of bile duct was not shown in TG-101, previous studies showed that higher dose radiation of bile duct was related with higher risk of stenosis. Barney et al. [34] reported one case of grade 3 biliary stenosis after SBRT where the patient was treated with a dose of 50Gy in five fractions. Takahisa et al. [35] showed that the true threshold for biliary stenosis would be between 40Gy in 5 fractions and 80Gy in 5 fractions, and a special caution was necessary when treating patients with more than 40Gy in 5 fractions. Before this study, we had 2 cases with obstructive jaundice after 50Gy/5f and 54Gy/6f, and their PET-CT showed bile duct stenosis but no active lesion. Since then, we adopted the dose fraction of $49 \mathrm{~Gy} / 7 \mathrm{f}$ and $48 \mathrm{~Gy} / 8 \mathrm{f}$ in SBRT of small HCC when the tumor is near to bile duct to reduce this risk. And until now, no patient has had bile duct stenosis by the two doses and fraction sizes above, so we consider they were safe fractionated regimens. Considering both the relation of BEDs and survival rates in our study, we think the trade-off between the efficacy and risk of SBRT in tumors near the bile duct deserves further study, and the optimal dose fractionated regimens needs large-sized samples to explore.

This study has several limitations. First, this was a retrospective study. Second, the number of patients in the two groups is much different, which may cause bias. A relevant prospective clinical trial is in progress (Clinicaltrails NCT 03295500). We hope to get objective and accurate results.

\section{Conclusion}

SBRT is a safe and effective option for CP-A HCC patients, and an increased $\mathrm{BED}_{10}$ and lower $\mathrm{CP}$ score are associated with improved OS and PFS. The results indicate that a strategy escalating radiation doses over a limited time frame are worth exploring in a prospective clinical trial. Meanwhile, PLT count should be considered, which is a predictive factor of RILD in SBRT of HCC.

\section{Additional file}

Additional file 1: The information of the 108 patients involved in the study. (XLSX $15 \mathrm{~kb}$ )

\section{Abbreviations}

AFP: Alpha fetoprotein; ALP: alkaline phosphatase; BED: Biologically effective dose; Cls: Confidence intervals; CP: Child-Pugh; CR: Complete response; $C T$ : computed tomography; DMFS: distant metastasis-free survival; ECOG: Eastern cooperative oncology group; GTV: Gross target volume; HCC: Hepatocellular carcinoma; HRs: Hazard ratios; LC: Local control; MRI: Magnetic resonance imaging; OS: Overall survival; P: P-value; PD: Progressive disease; PET-CT: Positron emission tomography-computed tomography; PR: Partial response; PTV: Planning target volume; RFA: Radiofrequency ablation; RILD: Radiation-induced liver disease; SBRT: Stereotactic body radiation therapy; SD: Stable disease

\section{Acknowledgements}

We appreciate Ye Lin (University of Chinese Academy of Sciences) for her valuable suggestions and editing support.

\section{Authors' contributions}

JS and TZ involved in drafting the manuscript and revising it critically for important intellectual content, and JS, WH and JD made substantial contributions to analysis and interpretation of data; JW, AZ and DL made substantial contributions to acquisition of data; WL and DZ were responsible for follow up content. XD was responsible for the final approval of the version to be published. Each author has participated sufficiently in the work to take public responsibility for appropriate portions of the content; and agreed to be accountable for all aspects of the work in ensuring that questions related to the accuracy or integrity of any part of the work are appropriately investigated and resolved.

\section{Funding}

This study was supported by grants from Beijing Municipal Science and Technology Commission Fund (Z171100001017181) whose responsible person was Wengang Li, who was responsible for follow up content.

\section{Availability of data and materials}

The information of the 108 patients involved in this study is presented in the Additional file 1

Date sharing is not applicable to this article as no datasets were generated or analyzed during the study.

\section{Ethics approval and consent to participate}

This study was approved by the Institutional Review Board of 302 Hospital of PLA (People's Liberation Army) and was conducted in accordance with the Declaration of Helsinki and internationally accepted ethical guidelines.

During their admission for SBRT treatment, the patients enrolled in this study 
provided written informed consent for their information to be stored in hospital databases and used for research.

\section{Consent for publication}

Not Applicable.

\section{Competing interests}

The authors declare that they have no competing interests.

Received: 26 February 2019 Accepted: 19 August 2019

Published online: 28 August 2019

\section{References}

1. Torre LA, Bray F, Siegel RL, Ferlay J, Lortet-Tieulent J, Jemal A. Global cancer statistics, 2012. Ca A Cancer J Clinicians. 2015;65(2):87-108.

2. Chen W, Zheng R, Baade PD, Zhang S, Zeng H, Bray F, Jemal A, Yu XQ, He J. Cancer statistics in China, 2015. CA Cancer J Clin. 2016;66(2):115-32.

3. Huertas A, Baumann AS, Saunier-Kubs F, Salleron J, Oldrini G, Croisã-Laurent V, Barraud H, Ayav A, Bronowicki JP, Peiffert D. Stereotactic body radiation therapy as an ablative treatment for inoperable hepatocellular carcinoma. Radiother Oncol. 2015;115(2):211-6.

4. Su TS, Liang P, Lu HZ, Liang J, Gao YC, Zhou Y, Huang Y, Tang MY, Liang JN. Stereotactic body radiation therapy for small primary or recurrent hepatocellular carcinoma in 132 Chinese patients. J Surg Oncol. 2016;113(2):181-7.

5. Andolino DL, Johnson CS, Maluccio M, Kwo P, Tector AJ, Zook J, Johnstone PA, Cardenes HR. Stereotactic body radiotherapy for primary hepatocellular carcinoma. Int J Radiat Oncol Biol Phys. 2011;81(4):e447-53.

6. Su TS, Liang P, Liang J, Lu HZ, Jiang HY, Cheng T, Huang Y, Tang Y, Deng X. Long-term survival analysis of stereotactic ablative radiotherapy versus liver resection for small hepatocellular carcinoma. Int J Radiat Oncol Biol Phys. 2017;98(3):639.

7. Wahl D, Stenmark M, Tao Y, Pollom E, Caoili E, Lawrence T, Schipper M, Feng $\mathrm{M}$. Outcomes after stereotactic body radiotherapy or radiofrequency ablation for hepatocellular carcinoma. J Clin Oncol. 2016;34(5):452-9.

8. Stahl JM, Ross R, Harder EM, Mancini BR, Soulos PR, Finkelstein SE, Shafman TD, Dosoretz AP, Evans SB, Husain ZA. The association between biologically effective dose (BED) and radiation treatment schedule on overall survival in stage I non-small cell lung cancer (NSCLC) patients treated with stereotactic body radiation therapy (SBRT). Int J Radiat Oncol Biol Phys. 2016;96(5):1011-20.

9. Z Zhu L, Zhang S, Xu X, Wang B, Wu K, Deng Q, Xia B, Ma S. Increased biological effective dose of radiation correlates with prolonged survival of patients with limited-stage small cell lung Cancer: a systematic review. PLoS One. 2016;11(5):e0156494.

10. Beskow C, Ågrencronqvist AK, Lewensohn R, Tomadasu I. Biological effective dose evaluation and assessment of rectal and bladder complications for cervical cancer treated with radiotherapy and surgery. J Contemp Brachytherapy. 2012;4(4):205-12.

11. Velec M, Haddad CR, Craig T, Wang L, Lindsay P, Brierley J, Brade A, Ringash J, Wong R, Kim J. Predictors of liver toxicity following stereotactic body radiation therapy for hepatocellular carcinoma. Int J Radiat Oncol Biol Phys. 2017;97(5):939.

12. Jung J, Yoon SM, Kim SY, Cho B, Park JH, Kim SS, Song SY, Lee SW, Ahn SD, Choi EK. Radiation-induced liver disease after stereotactic body radiotherapy for small hepatocellular carcinoma: clinical and dose-volumetric parameters. Radiat Oncol. 2013:8(1):1-7.

13. Murray $L J$, Dawson LA. Advances in stereotactic body radiation therapy for hepatocellular carcinoma. Semin Radiat Oncol. 2017;27(3):247.

14. European oF, R, Treatment Of C, Llovet J, Ducreux M, Lencioni R, Bisceglie AD, Galle P, Dufou J, Easleortc C: European association for the study of the liver, European organisation for research and treatment of Cancer. EASLEORTC clinical practice guidelines: Management of hepatocellular carcinoma 2012.

15. Johnson PJ, Berhane S, Kagebayashi C, Satomura S, Teng M, Reeves HL, O'Beirne J, Fox R, Skowronska A, Palmer D. Assessment of liver function in patients with hepatocellular carcinoma: a new evidence-based approach-the ALBI grade. J Clin Oncol. 2015;33(6):550-8.

16. Benedict SH, Yenice KM, Followill D, Galvin JM, Hinson W, Kavanagh B, Keall P, Lovelock M, Meeks S, Papiez L. Stereotactic body radiation therapy: the report of AAPM task group 101. Med Phys. 2010;37(8):4078-101.

17. Jones B, Dale RG, Deehan C, Hopkins KI, Morgan DA. The role of biologically effective dose (BED) in clinical oncology. Clin Oncol. 2001;13(2):71-81.
18. Fowler JF. The linear-quadratic formula and progress in fractionated radiotherapy. Br J Radiol. 1989;62(740):679-94.

19. Cox JD, Stetz J, Pajak TF. Toxicity criteria of the radiation therapy oncology group (RTOG) and the European organization for research and treatment of cancer (EORTC). Int J Radiat Oncol Biol Phys. 2015;31(5):1341-6.

20. Lawrence TS, Robertson JM, Anscher MS, Jirtle RL, Ensminger WD, Fajardo LF. Hepatic toxicity resulting from cancer treatment. Int J Radiat Oncol Biol Phys. 1995;31(5):1237.

21. Liang SX, Huang XB, Zhu XD, Zhang WD, Cai L, Huang HZ, Li YF, Chen L, Liu $M Z$. Dosimetric predictor identification for radiation-induced liver disease after hypofractionated conformal radiotherapy for primary liver carcinoma patients with child-Pugh grade a cirrhosis. Radiother Oncol. 2011;98(2):265-9.

22. Cheng JC, Wu JK, Lee PC, Liu HS, Jian JJ, Lin YM, Sung JL, Jan GJ. Biologic susceptibility of hepatocellular carcinoma patients treated with radiotherapy to radiation-induced liver disease. Int J Radiat Oncol Biol Phys. 2004;60(5): 1502-9.

23. Pan CC, Kavanagh BD, Dawson LA, Li XA, Das SK, Miften M, Ten Haken RK. Radiation-associated liver injury. Int J Radiat Oncol Biol Phys. 2010; 76(3):S94-S100

24. Chapman TR, Bowen SR, Schaub SK, Yeung RH, Kwan SW, Park JO, Yu L, Harris WP, Johnson GE, Liou IW. Toward consensus reporting of radiationinduced liver toxicity in the treatment of primary liver malignancies: Defining clinically relevant endpoints. Pract Radiat Oncol. 2018;8(3):157-66.

25. Zhang T, Sun J, He W, Li H, Piao J, Xu H, Duan X. Stereotactic body radiation therapy as an effective and safe treatment for small hepatocellular carcinoma. BMC Cancer. 2018;18(1):451.

26. Ueno M, Hayami S, Shigekawa Y, Kawai M, Hirono S, Okada Kl, Tamai H, Shingaki N, Mori $Y$, Ichinose M. Prognostic impact of surgery and radiofrequency ablation on single nodular $\mathrm{HCC} \leqslant 5 \mathrm{~cm}$ : cohort study based on serum HCC markers. J Hepatol. 2015;63(6):1352-9.

27. Ohri N, Tomé WA, Méndez Romero A, Miften M, ten Haken RK, Dawson LA, Grimm J, Yorke E, Jackson A. Local control after stereotactic body radiation therapy for liver tumors. Int J Radiat Oncol Biol Phys. 2018.

28. Lee $Y$, Auh S, Wang Y, Burnette B, Wang Y, Meng Y, Beckett M, Sharma R, Chin $\mathrm{R}$, Tu T, et al. Therapeutic effects of ablative radiation on local tumor require CD8+ T cells: changing strategies for cancer treatment. Blood. 2009; 114(3):589-95.

29. Schaue D, Ratikan JA, Iwamoto KS, Mcbride WH. Maximizing tumor immunity with fractionated radiation. Int J Radiat Oncol Biol Phys. 2012; 83(4):1306-10.

30. Wang L, Feng Y, Ma X, Wang G, Wu H, Xie X, Zhang C, Zhu Q. Diagnostic efficacy of noninvasive liver fibrosis indexes in predicting portal hypertension in patients with cirrhosis. PLoS One. 2017;12(8):e0182969.

31. Kim MY, Jeong WK, Baik SK. Invasive and non-invasive diagnosis of cirrhosis and portal hypertension. World J Gastroenterol. 2014;20(15):4300-15.

32. Nozaki R, Murata S, Nowatari T, Maruyama T, Ikeda N, Kawasaki T, Fukunaga K, Ohkohchi N. Effects of thrombopoietin on growth of hepatocellular carcinoma: is thrombopoietin therapy for liver disease safe or not? Hepatol Res. 2013;43(6):610-20.

33. Liang SX, Zhu XD, Xu ZY, Zhu J, Zhao JD, Lu HJ, Yang YL, Chen L, Wang AY, Fu XL. Radiation-induced liver disease in three-dimensional conformal radiation therapy for primary liver carcinoma: the risk factors and hepatic radiation tolerance. Int J Radiat Oncol Biol Phys. 2006;65(2):426-34.

34. Barney BM, Haddock MG, Miller RC, Olivier KR. Clinical outcomes and toxicity using stereotactic body radiotherapy (SBRT) for advanced cholangiocarcinoma. Radiat Oncol. 2012;7(1):67.

35. Takahisa E, Atsuya T, Naoko S, Yohei O, Yousuke A, Naoyuki S, Etsuo K. Acceptable toxicity after stereotactic body radiation therapy for liver tumors adjacent to the central biliary system. Int J Radiat Oncol Biol Phys. 2013; 85(4):1006-11.

\section{Publisher's Note}

Springer Nature remains neutral with regard to jurisdictional claims in published maps and institutional affiliations. 